\title{
Eliminating the Health Disparity of the Diabetic Foot: A Con- tinuing Public Health Crisis for African Americans and Latinos
}

\author{
Jacqueline E Sharpe, PhD, RN, CHES* \\ School of Nursing, Hampton University, Hampton, Virginia, United States of America
}

*Corresponding author: Jacqueline E Sharpe, PhD, RN, CHES, School of Nursing, Hampton University, Hampton, Virginia, United States of America

\section{Introduction}

According to the International Diabetes Federation 2020, it has been estimated that 463 million people have diabetes and that the total number of people with diabetes is predicted to rise to 578 million by 2030 and to 700 million by 2045 [1]. "Diabetes mellitus is a chronic disorder of glucose metabolism with serious clinical consequences. The multi-system complications of diabetes include microvascular (retinopathy, nephropathy, neuropathy) and macrovascular (ischemic heart disease, stroke, peripheral vascular disease) endpoints" [2]. One of the most important complications of diabetes is the diabetic foot ulcer which if not treated leads to infection, deep tissue necrosis, and limb amputation [3]. Approximately, $80 \%$ of diabetes-related lower extremity amputations are preceded by a foot ulcer $[4,5]$. Every 30 seconds, a foot amputation happens because of diabetes in the world, and more than one million patients lose their diabetic foot annually due to the disease [6]. Recent research conducted on the diabetic ulcer conclude that $75 \%$ of foot ulcers are preventable, but prevention receives little attention [7-9]. The existence of racial and ethnic disparities in leg amputations is well documented [5]. It is without doubt that diabetic foot care is a public health problem [10]. The aim of this paper is to maintain attention to the plight of prevention of the diabetic foot ulcer, particularly for those racial/ ethnic minority persons who must not be overlooked for healthcare measures that can save their lives, and for healthcare providers to not allow ones inability to pay be a rejection for not preserving that life and quality of life. The release of the 2020 National Diabetes Sta- tistics Report for the United States continues to show that the ethnic minority groups, age 18 or older, continues to have the highest prevalence of diagnosed diabetes. Healthcare professionals do harm when nothing is done, being an advocate for preserving life does not cost, it creates value.

\section{The Epidemiology of Diabetes}

Epidemiology involves the study of the distribution and determinants of health-related states or events in human populations, and the application of this study to prevent and control health problems [11]. Diabetes is part of the multifactorial etiology in chronic disease epidemiology, prevention and control of diseases, disorders, injuries, disabilities, and deaths [11]. Diabetes was the seventh cause of death in the United States in 2017. Diabetes mellitus is a chronic disease and is one of the leading causes of limb loss, affecting now 463 million people worldwide. The relationship between diabetes and obesity is of key interest to population health stakeholders and healthcare professionals supporting diabetes prevention and management initiatives within their communities [12]. Most Disparities in leg amputation are attributable to complex interaction of policy factors, such as access to healthcare and modes of healthcare finance; institutional and provider factors, such as experience, use of guidelines, stereotyping and biases; and patient factors such as compliance and medical seeking behavior [13]. Most epidemiological work on diabetes has used datasets designed for other purposes; major racial and ethnic disparities in health are well documented for the United States of America [14].

Citation: Sharpe JE (2020) Eliminating the Health Disparity of the Diabetic Foot: A Continuing Public Health Crisis for African Americans and Latinos. Int J Diabetes Clin Res 7:120. doi.org/10.23937/23773634/1410120

Accepted: April 28, 2020: Published: April 30, 2020

Copyright: (C) 2020 Sharpe JE. This is an open-access article distributed under the terms of the Creative Commons Attribution License, which permits unrestricted use, distribution, and reproduction in any medium, provided the original author and source are credited. 
Projection of the future diabetes burden in the United States through 2060 was conducted and projected that racial disparities in the diabetes burden would get worse if no action was taken and that approximately one in four Blacks would have diagnosed diabetes by the year 2060 [15]. Diabetes affect the ethnic minority groups in the worst ways, not just the disease itself, but also in all of the complications. Geographical location in the US, also known as the 'Diabetes Belt,' in addition to community factors, play a role in the increasing prevalence of obesity and diabetes [16].

\section{The Diabetic Foot Health Disparity}

"A health disparity is defined as a particular type of health difference that is closely linked with social, economic, and/or environmental disadvantage. Health disparities adversely affect groups of people who have systematically experienced greater obstacles to health based on their racial or ethnic group; religion; socioeconomic status; gender; age; mental health; cognitive; sensory or physical disability; sexual orientation or gender identity; geographical location, or other characteristics historically linked to discrimination or exclusion" [17]. The existence of racial and ethnic disparities in leg amputations rates is well documented [13]. In patients with diabetes, foot amputations among Black people have been historically higher compared with White people [18]. African Americans (Black people) are at higher risk for two diseases -diabetes and peripheral arterial disease (PAD)-that together threaten to cause them more preventable amputations than other populations [19].

There is a dramatic rise in the prevalence of Type 2 diabetes Mellitus and its associated complications across the globe [20]. In the United States, according to the Center for Disease Control (CDC) 2020 National Diabetes Statistics Report, the percentage of age-adjusted incidence of diagnosed diabetes among adults aged 18 years or older in the United States during 2017-2018, is 5.2 for White, 8.2 for non-Hispanic Black, and 9.7 for Hispanic [20]. "US Hispanics/Latinos are disproportionately affected by diabetes compared with non-Latino Whites and previous work has shown that the prevalence of diabetes related cognitive deterioration is higher in Hispanic/Latinos than in non-Latino Whites" [21]. In the US mainland, another study showed that Latinos have a high rate of type 2 diabetes mellitus and exhibit poor glycemic control, high rates of complications, poor outcomes, and diminished quality of life [22]. Medicare Advantage enrollees in the territory of Puerto Rico receive substantially lower quality of care than white or Hispanic enrollees residing in the United States [23]. Rates of diabetes are generally higher among African Americans and Hispanics than among non-Hispanic Whites, and rates have been reported to be $25 \%$ higher among Hispanics than among African Americans [24]. New diabetes cases were higher among non-Hispanic blacks and people of Hispanic origin than non-Hispanic Asians and
non-Hispanic whites [20]. As the prevalence of diabetes increases, so does the opportunity for complications of diabetes to exist.

Research in recent years has shown distinct ethnic differences in the prevalence of diabetic complications, including amputation and more recently, foot ulceration [25]. A US News Report in 2014 revealed significant racial and regional disparities for diabetes care in the US; it reported that amputation rates for Black patients with diabetes and peripheral arterial disease in the rural Southeast can be as high as seven times the rate of other regions [26]. It is a health disparity for the Latinos, African Americans and Native Americans because they are the people suffering the increasingly worst outcomes for this disease-death and disabilities and meet the definition of a health disparity since a health disparity is an inequality that occur in the provision of healthcare and access to healthcare across different racial, ethnic and socioeconomic groups [27]. Dr. Soto de Laurido, Health Disparities, p. 5. Racial and ethnic minorities are disproportionately affected by diabetes, are more likely to have poorly controlled diabetes, and area at significantly greater risk of experiencing poor diabetes-related outcomes than are Whites.

In the United States, current 2020 data from the Center of Disease Report reveals that "New diabetes cases were higher among non-Hispanic Blacks and people of Hispanic origin than non-Hispanic Asians and non-Hispanic whites". Incidence is the rate of new cases of diagnosed diabetes among adults in the United States that went down in 2015 and had gone down each year since 2008. The diabetes epidemic continues to grow at an alarming rate [20]. Diabetes prevalence is the total number of existing cases, including new cases among adults and it is continuing to go up; the prevalence for diabetes for 2020 is now increased from 30.3 million in 2015 people to now 34.2 million people [20]. The projected number and percent of adults with diagnosed diabetes would increase from 22.3 million in 2014 to 39.7 million in 2030 to 60.6 million in 2060 [20]. The percentage of adults with diabetes increased with age, reaching $26 \%$ among those aged 65 years or older [20] in those $>20$ years of age are $7.6 \%$ for both Cubans and for Central and South Americans, 13.3\% for Mexican Americans and $13.8 \%$ for Puerto Ricans. $18.7 \%$ of all African Americans $\geq 20$ years of age have diagnosed or undiagnosed diabetes [20]. Even before Hurricane Maria brought widespread devastation to the island, Puerto Rico had a diabetes prevalence rate about $50 \%$ higher than the general US population [28].

A recent report from the United Kingdom (UK) revealed that Type 2 diabetes is a major UK public health priority and among minority ethnic communities, the prevalence is alarmingly high, approximately three to five times higher than in the White British population. It is well documented that minority ethnic groups in 
the UK and in other regions of the world suffer disproportionately from diabetes compared with nonminority populations [29]. A current literature review on the influence of race, ethnicity and social determinants of health on diabetes outcomes concluded that in addition to being skilled in assessing social determinants of health, this professional group found that "more research is needed to identify the separate and combined influence of race and ethnicity and social determinants of health on process of care, quality of care, and outcomes in diabetes, especially in the South, where the burden of disease is particularly high" [30]. The existence of racial and ethnic disparities in leg amputations rates is well documented [13]. The diabetic foot is usually listed in the review of literature as a complication of diabetes, but not as a health disparity. There is a recent study of 2019 conducted at Central Michigan University, College of Medicine that researched "to confirm disparities in diabetes mortality rates based on race, determine if race predicts combinations of diabetes and multiple chronic conditions (MCC) that are leading causes of death, and determine if combinations of diabetes plus MCC mediate the relationship between race and mortality" [31]. This study was a retrospective cohort study of 443, 932 Medicare beneficiaries in the state of Michigan. The Medicare group is the group of the age of 65 and older, and diabetes affect this group in larger numbers of the population.

Another study carried out using a large data set was conducted by Tan Tze-Woel and others (2019) [32]. This study was conducted over a 3-year period, from 20022015. It is noteworthy to point out that the purpose of this study was to evaluate the disparities in the outcomes of Whites, $(98,361)$ African Americans $(24,583)$, and Hispanic $(24,472)$ and Native American, $(1,654)$ patients admitted in the hospital with diabetic foot ulcers. These researchers used the Nationwide Inpatient Sample (NIS) retrospectively to identify the patients in the study. This inpatient sample was part of the Healthcost and Utilization Project. The findings of this study concluded that even though there has been an overall reduction in diabetes-related lower extremity amputations in the US and the world, there simultaneously has been an increase in use of aggressive toe and feet amputations to control the foot infection that was observed in all groups, including for Whites as well. These authors also pointed out that one possible reason for the reduction in major amputations was the increase in the revascularization procedures for these types of patients. What may not have been an area of exploration for these researchers is knowledge of the Black culture. There is still some distrust of the American Health Care System from past historical events and there is belief in this group not to seek medical attention if in the lower socio-economic group. It is well known that those who are in a higher socioeconomic group have access to a higher level of healthcare. The doctor-patient relationship is also an area of possibilities for outcomes that are not mentioned.

The role of healthcare access and socioeconomic status also plays a very important role in healthcare. It is well known by all persons that the quality of care received depends on cost and available resources. A study conducted at Meharry Medical College in 2018 used a sample size of 2,172 participants from the 2013 Medical Expenditure Panel Survey that included data of adults diagnosed with Type 2 diabetes [33]. This study concluded that lack of insurance coverage and education explained some of the racial/ethnic disparities observed in diabetes quality of care and improving quality of diabetes care could help to reduce rates of diabetes complications, healthcare costs and mortality. The international community has also been very actively engaged in researching and writing about the diabetic foot and conclusions are similar and consistent with other countries for the past decade. Diabetic foot care in other countries has also been concluded as a public health problem with devastating human, social and economic consequences [10]. Such as in Pakistan, where the authors write that "foot disease is considered to be one of the most common complications of diabetes mellitus and the diabetic foot refers to a spectrum of disease. Foot complications from diabetes are one of the main causes of amputation and its subsequent physical and emotional problems" [10].

\section{Two Ethnic Minority Groups of Concern}

Latinos are the largest ethnic minority group in America, this group in 2019 was $18.3 \%$ of the population of the population [34]. The review of literature on the health care issues and health problems of this group is lacking and for the most part, outdated. Data found is either about Latinos. The most current data for this group is from the Center for Disease Control (CDC) 2020 report. The CDC 2020 report shows interesting findings regarding the total diabetes percentage for the race/ ethnicity groups: 11.9 white (non-Hispanic), 16.8 for Black (non-Hispanic), 14.9 Asian (non-Hispanic), and Hispanic, 17.9. The Asian group's number has increased from $8.0 \%$ (2013-2015 data) to that now of 16.0 [34]. Information found will be that about Latinos in California, Texas, or Puerto Rico. Key findings of this report state that, "New diabetes cases were higher among non-Hispanic Blacks and people of Hispanic origin than non-Hispanic Asians and non-Hispanic Whites". If the number of cases of diabetes is steadily increasing in the ethnic/ racial minority groups, one can assume that the complications of diabetes with this group will also increase. Being that the racial/minority groups are also the lower socioeconomic groups without health insurance or inadequate insurance, complications will also increase [35]. Healthcare is not free in the United States and ethnic minorities have gone from hardly any healthcare as a group of people to now some health care, depending 
on one's income. Healthcare is a business and one must pay. If one cannot afford to purchase, one does not get the same care as the higher income group. Those in the international community who are similar in economic levels share the same fate.

Research pertaining to the diabetic foot ulcer is ongoing. Many of the present bench research focuses on drug therapy, surgery, glucose levels, and hyperbaric oxygenation and various techniques for wound healing. Despite of all previous knowledge, morbidity and mortality of diabetes, this disease has not improved sufficiently. A review of the literature of 1,443 articles was done by Bonner, Foster, and Spears-Lanoix to compile and evaluate published evidence for increasing foot care knowledge and self-care practices as part of a targeted diabetes mellitus type, 2-foot care intervention [36]. The findings from this study is noteworthy. These authors of this study concluded that "when adjusting for multiple chronic conditions, mortality disparities observed between Whites, Black/African Americans, and American Indians/Alaska Natives persist". This study concluded that "interventions and research studies that aim to reduce lower extremity complications are still lacking".

Advocacy efforts for the diabetic persons include the American Diabetes Association that includes its minority populations, and federal legislation such as the EDDPAC, the Eliminating Disparities in the Diabetes Prevention, Access and Care Act, the Special Diabetes Program, the Center for Disease Control, and the Patient Protection and Affordable Care Act of 2010. There are organizations that function to take care of the feet with publications about taking care of the feet, the National Diabetes Education Program and the International Diabetes Federation. All the knowledge of the research being conducted is remaining at levels 1 to 2 of the translational model. We need to move now to level 3 and 4 . As the numbers of people diagnosed by diabetes increase, there is a greater need to reach greater numbers of people who can avoid complications of diabetes. Even though technology has improved communication, professionals can add a humane touch when a person is diagnosed with a disease that will cause life changes. The community touch is lacking in many of the areas of the mainland of the US. One of the preventive measures mentioned by all researchers are preventive measures to be taken to prevent the possible complication of the diabetic foot. Medical care should always be part of any patient care.

What is now needed is application of principles of translational research as reviewed by Garst and others [37] in sustaining diabetes prevention and care intervention: A multiple case study of translational research projects. Eleven translational research projects were supported by the BRIDGES (Bringing Research in Diabetes to Global Environments and Systems) project of the International Diabetes Federation. Of the eleven projects done internationally, two were done in the United States. One project done in the United States was pertaining to preventing diabetes complications and focused on 'nonvisual foot inspection for people with visual impairment'. That study was short termed and now completed. There was another short-term project in the US done that was pertaining to motivational interviewing. Isabel Garcia de Quevedo and others [38] wrote about the challenges in real-life diabetes translation research: Early lessons from BRIDGES projects.

\section{A Need for More Preventive Work}

Of course, preventive action will not be the only solution. It is very evident that it will take diabetes self-management education and training that has been defined as "the process of facilitating the knowledge, skill, and ability necessary for diabetes self-care" [39]. Preventive work is important because it will help to save lives, and to prevent pain, and suffering of the newly diagnosed diabetic. Many persons diagnosed with diabetes view their diagnosis as a quick way to start losing functioning of all bodily organs and feel neglected because no one seem to have time to talk to them, but just enough time to hand out a booklet or web site to be viewed. We are missing a need for more of the humane touch in dealing with the newly diagnosed diabetic patient. A health sciences academic environment can be very supportive of providing educational services and emotional support to diabetics. There are pharmacy majors, nursing majors, politicians, podiatrists, physicians, health departments, exercise fitness majors, and physical therapy majors, all at some universities to create an educational intervention for newly diagnosed diabetics. Involvement with the community churches, fast food stores, and grocery stores are part of the community as well. There is a need to help diabetics to evaluate their community and decide what actions and behaviors are most beneficial to themselves and their families. There is mention of an integrated community diabetes model [40]. Other writers have written about different aspects emphasizing Latino care and issues. There is an abundance of research done about wound care of the diabetic foot ulcer and the conclusions have stated continually that prevention is key for the self-management of diabetes for the patient. Bus and van Netten have stated that prevention thereof receives little attention [33]. Recent self-management research is mostly qualitative and large datasets are used for reviewing information about the ethnic minorities for having a statistical meaningful sample size.

A study by Tommy Chiou and others conducted a study on trends in racial and ethnic disparities in diabetes related complications from 1997-2017 [41]. They, too, used a large dataset of patients that consisted of non-Hispanic 31,722 Whites, 11,020 Blacks, and 9389 
Hispanics. Their study demonstrates the "importance of examining trends by racial/ethnic groups to discern the extent to which the documented improvements have been distributed equitably and to identify opportunities to improve care for selected diabetes populations". What these studies have all pointed out is the need for community-based research studies. What also that has been missing from these studies is the cultural influences in the lives of the ethnic minorities on a larger sample size. Small sample sizes cannot continue to be the norm method of research, nor using just large data sets for analysis. The African American and the Latino group of people are strongly influenced by their culture, and getting their communities involved is a must. Telling these what to do is meaningless if the culture of the community is not supportive. Just human to human caring being demonstrated is a component worth further research. A health professional's knowledge and caring attitude is instrumental to patient compliance and good health. Much work has already been done on this topic, but this year's data of 2020 is not decreasing for diabetes, the data for diabetes for the minority groups still continue to rise. Therefore, it is time for researchers to reassess methods previously used and set forth newer strategies that will enable these people to live and to enjoy life.

\section{References}

1. (2020) International Diabetes Federation.

2. Forouhi N, Wareham N (2010) Epidemiology of diabetes medicine. 38: 602-606.

3. Padma K, Arundathi S, Indira S (2016) A study to assess the knowledge on foot care among diabetic client's endocrinology OPD in Narayana medical college and hospital, Nellore. International Journal of Medicine Research 1: 87-90.

4. Obeid M, Moughames E, Aboulhosn P, Madi R, Farah M, et al. (2018) Epidemiology and susceptibility profiles of diabetic foot infections in five hospitals in Lebanon. J Infect Dev Countries 12: 347-351.

5. Barshes NR, Sigireddi M, Wrobel JS, Mahankali A, Robbins JM, et al. (2013) The system of care for the diabetic foot: Objectives, outcomes, and opportunities. Diabet Foot Ankle.

6. Fardazar F, Tahari F, Solhi M (2018) Diabetes and metabolic syndrome: Clinical research reviews. Science Digest 12: 853-858

7. Bus S, van Netten $J(2016)$ A shift in priority in diabetic foot care and research: $75 \%$ of foot ulcers are preventable. Diabetes Metab Res Rev 32: 195-200.

8. Phillips A, Mehl A (2015) Diabetes mellitus and the increased risk of foot injuries. J Wound Care 24: 4-7.

9. Bonner T, Guidry J, Jackson Z (2019) Association between foot care knowledge and practices among African Americans with type 2 diabetes: An exploratory pilot study. J Natl Med Assoc 111: 256-261.

10. Somroo J, Hashmi A, Iqbal Z, Ghori (2011) Diabetic foot care-a public health problem. J Medicine 12: 109-114.

11. Merrill RM (2021) Introduction to Epidemiology. ( $8^{\text {th }}$ edn), Jones \& Bartlett Learning, Burlington.
12. Reflections on Nursing Leadership (2017) The face of diabetes in the United States Gallup Share case research.

13. Barshes, Sharath S, Zamani N, Smith K, Serag H, et al. (2018) Racial and geographic variation in leg amputations among Texans. Tex Public Health J 70: 23-27.

14. McKinlay J, Marceau L (2000) US public health and the 21st century: Diabetes mellitus. Lancet 356: 757-761.

15. Ji Lin, Theodore J Thompson, Yiling J Cheng, Xiaohui Zhuo, Ping Zhang, et al. (2018) Projection of the future diabetes burden in the United States through 2060. Population Health Metrics 16: 9.

16. Myers CA, Slack T, Broyles ST, Heymsfield SB, Church TS, et al. (2017) Diabetes prevalence is associated with different community factors in the diabetes belt versus the rest of the United States. Obesity (Silver Spring) 25: 452-459.

17. Rose Patti (2018) Health disparities, diversity, and inclusion. JB Learning Company, Burlington.

18. Harris CM, Albaeni A, Thorpe RJ, Norris KC, Abougergi MS (2019) Racial factors and inpatient outcomes among patients with diabetes hospitalized with foot ulcers and foot infections, 2003-2014. PLoS One 14: e0216832.

19. Press Release (2016) African Americans, diabetes, and amputation trends. Society for Vascular Surgery.

20. Center for Disease Control (2020) National Diabetes Statistics Report.

21. Lemon S, Rosal M, Welch G (2011) Measuring quality of life in low-income, Spanish-speaking Puerto Ricans with type 2 diabetes residing in the mainland US. Qual Life Res 20: 1507-1511.

22. Ekfassy T, Aiello AE, Schneiderman N, Haan MN, Tarraf $W$, et al. (2019) Relation of diabetes to cognitive function in Hispanic/Latinos of diverse background in the United States. J Aging Health 31: 1155-1171.

23. Colon H, Sanchez-Cesareo M (2016) Disparities in health care in Puerto Rico compared with the United States. JAMA Intern Med 176: 794-795.

24. Tucker K, Bermudez O, Castaneda C (2000) Type 2 diabetes is prevalent and poorly controlled among Hispanic elders of Caribbean origin. Am J Public Health 90: 12881293.

25. Abbott C, van Schie C (2002) A review of ethnic differences in risk factors for diabetic foot ulcers. Podiatry Today 15 : 49-54.

26. Leonard K (2014) Amputation more likely for blacks. US News.

27. Soto de Laurido L (2019) Health Disparities. Blackboard.

28. Communication Team (2018) Diabetes in Puerto Rico: An update on a public health crisis, one year after Hurricane Maria. Project Hope.

29. Goff LM (2019) Ethnicity and type 2 diabetes in the UK. Diaber Med 36: 927-938.

30. Walker R, Williams J, Egede L (2016) Influence of race, ethnicity and social determinants of health on diabetes outcomes. Am J Med Sci 351: 366-373.

31. Clements J, West BT, Yaker Z, Lauinger B, McCullers D, et al. (2019) Disparities in diabetes-related multiple chronic conditions and mortality: The influence of race. Diabetes Res Clin Pract 159: 107984.

32. Tan T, Shih CD, Concha-Moore KC, Diri MM, Hu B, et al. (2019) Disparities in outcomes of patients admitted with diabetic feet infections. PLoS One. 
33. Canedo J, Miller ST, Schlundt D, Fadden MK, Sanderson M, et al. (2018) Racial/Ethnic disparities in diabetes quality of care: The role of healthcare access and socioeconomic status. J Racial Ethn Health Disparities 5: 7-14.

34. National Diabetes Statistics Report (2020) Estimates of diabetes and its burden in the United States.

35. Spencer MS, Rosland AM, Kieffer EC, Sinco BR, Valerio $M$, et al. (2011) Effectiveness of a community health worker intervention among African American and Latino adults with type 2 diabetes: A randomized controlled trial. Am J Public Health 101: 2253-2260.

36. Bonner T, Foster M, Spears-Lanoix E (2016) Type 2 diabetes-related foot care knowledge and foot self-care practice interventions in the United States: A systematic review of the literature. Diabet Foot Ankle 7: 29758.

37. Garst J, L'Heveder R, Siminerio LM, Motala AA, Gabbay $\mathrm{RA}$, et al. (2017) Sustaining diabetes prevention and care interventions: A multiple case study of translational research projects. Diabetes Res Clin Pract 130: 67-76.

38. Isabel Garcia de Quevedo, Linda Siminerio, Ronan L'Heveder, KM Venkat Narayan (2012) Challenges in real life diabetes translation research: Early lessons from BRIDGES projects. Diabetes Res Clini Pract 95: 317-325.

39. Carr D, Kappagoda M, Boseman L, Cloud LK, Croom B (2020) Advancing diabetes-related equity through diabetes self-management education and training: existing coverage requirements and considerations for increased participation. J Public Health Manag Pract 26: S37-S44.

40. Pastor M, Terriquez V, Lin M (2018) How community organizing promotes health equity, and how health equity affects organizing. Health Affairs 37: 358-363.

41. Chiou T, Tsugawa $Y$, Goldman D, Myerson R, Kahn M, et al. (2019) Trends in racial and ethnic disparities in diabetes-related complications 1997-2017. J Gen Intern Med 35: 950-951. 